\title{
The Method of the Energy Harvesting HEMS Router
}

\author{
Takashi Yoshikawa \\ Kindai University Technical College \\ Kasugaoka, Nabari, Mie 518-0459, JAPAN \\ yoshikawa@ktc.ac.jp
}

\begin{abstract}
In nowadays the energy management is the one of the biggest problems in the world. Especially the useless energy consumption is focused. In such circumstances HEMS (Home Energy Management System) is widely recognized as the useful technology for saving energy. On the other hand HEMS is never influenced around the common people. The reason is supposed coming from following three points. 1. High initial cost, 2. Location dependence (The present HEMS is mounted into the wall), 3. Personal unconcern for energy saving. Then we are trying to introduce the sensor network technology and energy harvesting technology into HEMS taking the place of the conventional wired system. It is because the energy harvesting can supply the power into sensor nodes automatically and never accompanies the labor of changing battery. So far we have developed the sensor network end node equipping the energy harvesting devices. Now I am trying to introduce the energy harvesting into routing nodes in order to aim the complete wireless system. In this paper I will describe the details how to design the scale of network and show the results of simulation.
\end{abstract}

Keywords: HEMS, Sensor Network, Energy Harvesting, Routing

\section{Introduction}

In recent days, the saving energy consumption is very important in the modern society. The major trend of the saving energy is focused on the industrial, factory, transport, electrical appliances and so on. On the other hand the almost all of the people in the developed country do not care the saving energy in their individual life. In such situation HEMS (Home Energy Management System) is very important technology. HEMS is the technology that the person monitors the amount of his energy consumption in a house, and tries to reduce the energy consumption. The people recognize HEMS is the built in system (wall mounted system) on the house. Then HEMS is regarded as expensive and lower flexibility for customize. This is because HEMS dose not spread to all the people.

Then we are trying to introduce the sensor network technology into HEMS. Thereby we can construct HEMS as the location free and cheep system. Moreover we are trying to introduce no battery system on sensor network.

That is based on using energy harvesting for the power source of the sensor network node. So far we have developed the HEMS with sensor network nodes on the star topology (Fig.1) and performed the trial test with good results ${ }^{(1)}$. In that trial the transmitting distance is restricted so short and affected by obstacles. Then we are developing routing node so as to extend the transmitting distance. So house is not such a big space, I have tried to adopt line topology on sensor network. Line topology is regarded as lower power consumption than mesh topology. In this study the most important thing is how to keep moving all sensor network nodes with energy harvesting. The validity of end node is proved in the previous our test. But the ability of the routing node is not clarified yet. So I have tried to make the communication protocol and define the data acquisition method to perform with lower energy consumption and estimated the network scale.


Fig.1. Sensor Network Topology 


\section{The Usable Average Power by Energy Harvesting in a House}

Sensor network end node is consists of various module shown in Fig.2. Every module keeps consuming the power. We have made the prototype of the sensor network end node and estimate the consumption power for each module (1). Every module does not always work because we use the sleep mode. Therefore we calculated the average power using the product of consumption power and duration time. The derived value varies according to the model of HEMS. HEMS model number means the index of the degree of application richness. Larger number of HEMS is more applicable. Table.1 shows the consuming average power of sensor network end nodes depending model of HEMS. In our first step, we are focusing model 4 HEMS is. Referring to the table.1, we have to compensate about $20[\mathrm{uW}]$ for consumption average power. Considering the convergence energy from physical energy to electric energy, the demanded power for circumference is estimated about $50[\mathrm{uW}]$.

Based on above considerations, we have measured the various harvesting energy in a house. We have measured illumination power generation with solar cell and vibration power generation with Piezo element by the human foot step (2) and temperature gradient power between human body and circumference with Perltier element and so on. Currently we can get the data that many kinds of energy harvesting in a house can generate more than $50[\mathrm{uW}]$, the larger one can generate more than $1[\mathrm{~mW}]^{(3)}$. Then now we regard that the usable average power is $50[\mathrm{uW}]$ and the

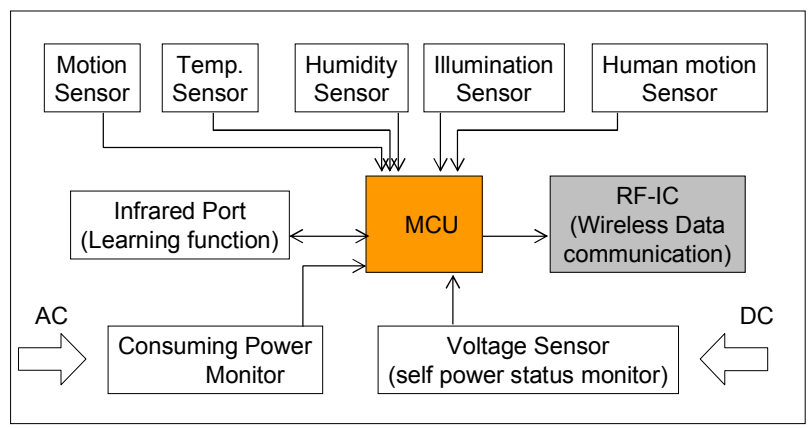

Fig.2. Sensor Network End Node Block Diagram

Table.1. The consumption Power of the End Node

\begin{tabular}{|c|c|c|c|c|c|}
\hline $\begin{array}{c}\text { Model 1 } \\
\text { Fixed }\end{array}$ & 7uW & $\begin{array}{c}\text { Model 2 } \\
\text { Fixed }\end{array}$ & 10uW & $\begin{array}{c}\text { Model 3 } \\
\text { Fixed }\end{array}$ & $10 u W$ \\
\hline $\begin{array}{c}\text { Model } 4 \\
\text { Fixed }\end{array}$ & $19 u W$ & $\begin{array}{c}\text { Model 5 } \\
\text { Fixed } \\
\text { Movable }\end{array}$ & $\begin{array}{c}19 u W \\
23 u W\end{array}$ & $\begin{array}{c}\text { Model 6 } \\
\text { Fixed } \\
\text { Movable }\end{array}$ & $\begin{array}{c}\text { 48uW } \\
35 u W\end{array}$ \\
\hline
\end{tabular}

consumption power of the sensor node is $20[\mathrm{uW}]$ under energy harvesting condition ${ }^{(4)}$.

\section{Introducing Routing nodes with Energy Harvesting}

Now we consider the consumption average power in the case of we use the routing node. Rooting node consumes the power of MCU module and RF-IC module in except for the sensor modules or other monitoring modules in Fig.2. The transmitting diagram is shown in Fig.3. As shown in Fig. 3 , the network has $\mathrm{n}$ router nodes and $\mathrm{k}$ end nodes. The routers are connected in a liner arrangement. In actual case in a house, the routers are arranged upper floor and lower floor and connected on the circumference wirelessly. Each routing node is synchronized by the master clock emitted from coordinator node sequentially.

The data time chart of this network is shown in Fig.5. Focusing the routing node closest to the coordinator node $\mathrm{R} 1$, when the $\mathrm{R} 1$ receives the master clock transmits the stacked data to the coordinator node and transmits the master clock to the lower routing node (R2), receives the stacked data from the lower routing node (R2), transmits the trigger pulse to the end nodes in its routing area,

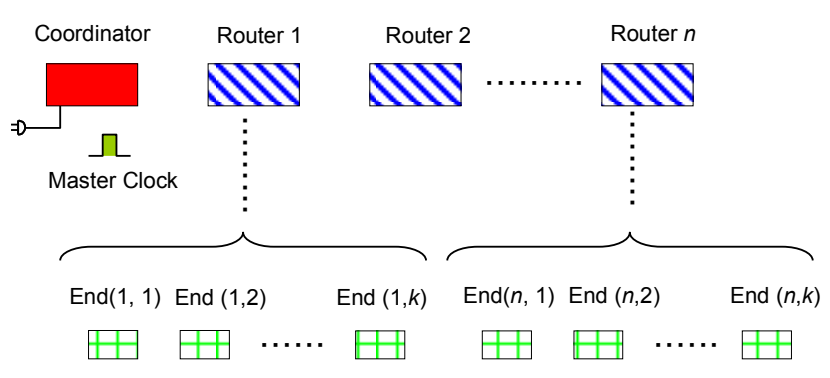

Fig.3. Data Transmitting Diagram

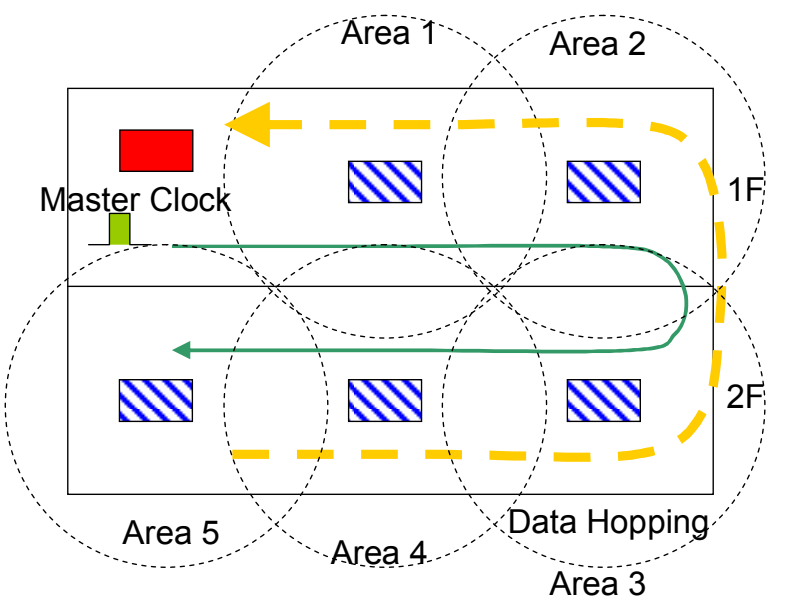

Fig.4. Data Hopping Sequence 


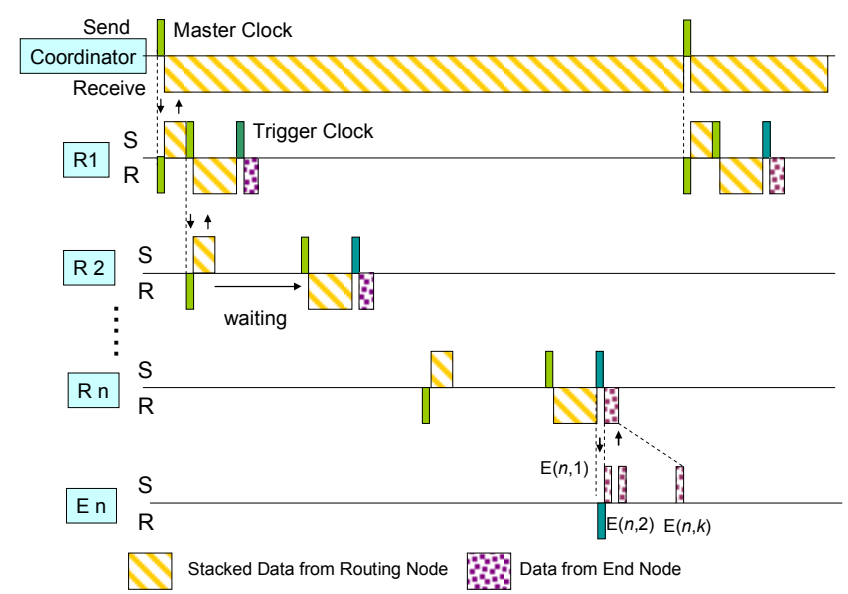

Fig.5. Data Time Flow of Routing Nodes for Sensor Network HEMS.

receives the data from each end nodes sequentially and stacks its data in the register. Focusing R2, after emitting stacked data to $\mathrm{R} 1$, prepares the waiting time for avoiding the data collision between the data emitted by E1 and stacked data emitted by R3.

The current consumption model of the sensor network node is measured and shown in Fig.6. The elements in Fig.6 are corresponding to the elements in Fig.5 (rewritten at the top of Fig.6). Here $n$ denotes the number of the routing nodes and $k$ denotes the number of the end nodes. The average consumption power is estimated using Fig.5 and Fig.6 with the applied voltage as 3 [V].

The data Transmit / Receive Procedure of the routing node is shown in Fig.7. Sending and Receiving packet has $n(n=4$ in Fig.7 example) blocks of data and each block of routing node contains $k$ blocks of the data of end nodes. Each routing node receives one packet containing $n$ ( $n=4$ in Fig. 7 example) blocks data from the lower router and transmits one packet containing $n$ ( $n=4$ in Fig. 7 example) blocks data

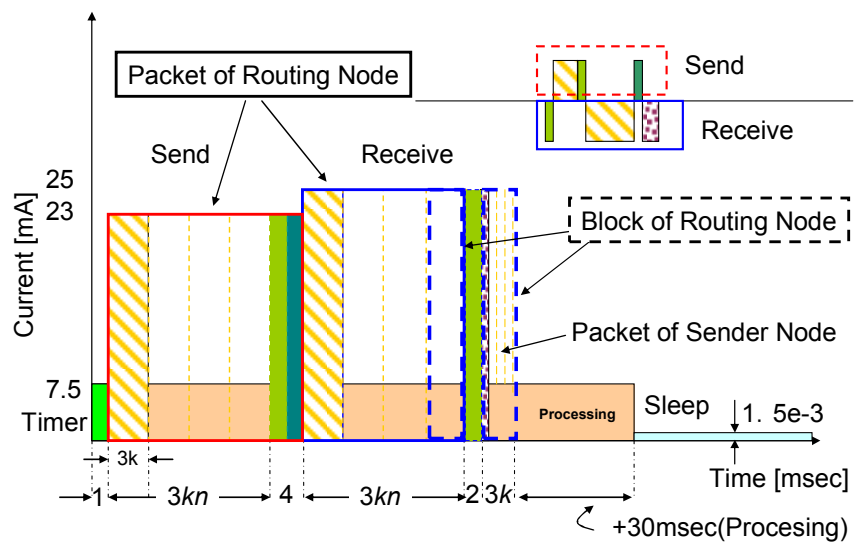

Fig.6. Current Consumption model of Routing Node

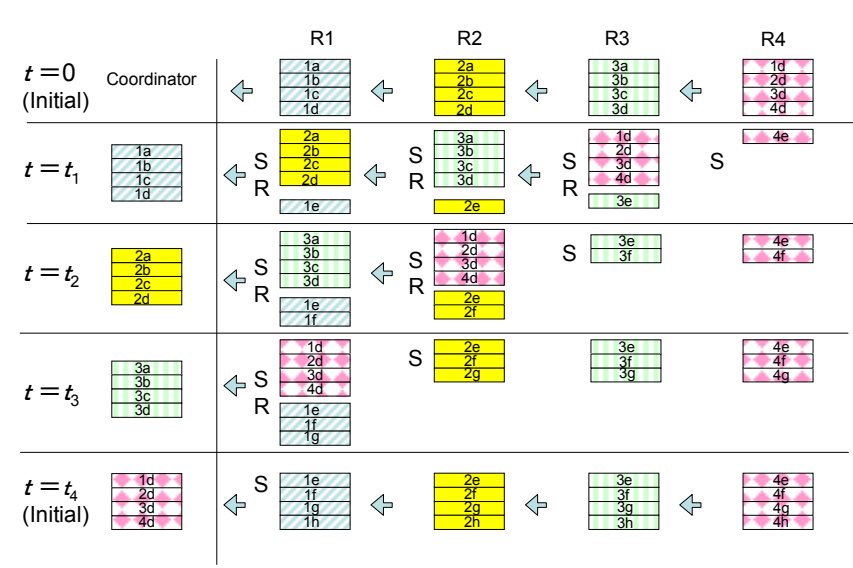

Fig.7. Routing Node Data Transmit / Receive Procedure

to the upper router (near the coordinator). As the result, the $n$ ( $n=4$ in Fig. 7 example) blocks data from all routing nodes arrives at coordinator in $n$ ( $n=4$ in Fig.7 example) time sequence. This way is very effective reducing the number of transmitting and receiving packets in addition to equivalently real-time data communication possible. On the other hand we have to pay attention that R1 needs 7 packets but R2 needs only one packet.

\section{Routing Topology}

\subsection{The Way for Revised Topology on small network}

In the session 3, it had been appointed that there are large differences in the number of times of transmitting and receiving between routing nodes. It leads to the result that we have to prepare the large amount of harvesting energy for each routing nodes because the largest one is dominant. Then I have considered other ways to reduce the maximum packets of routing node in a certain period. There are following two ideas under the condition that there are only two nearest nodes around the coordinator node assuming relatively small area network in a house.

(1) Two Line Data Acquisition (T.L.D.A.)

(2) Rotated Line Data Acquisition (R.L.D.A)

TLDA and RLDA are described in the case of $n=4$ in Fig.8,

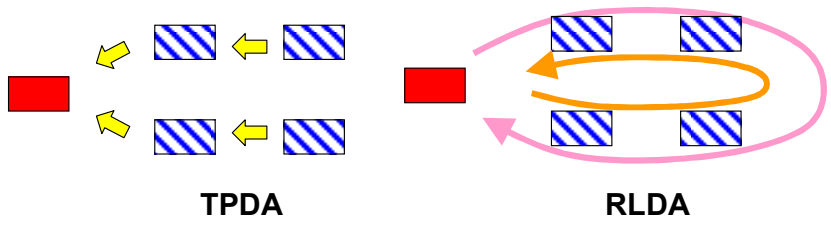

Fig.8. Data Acquisition Method for TLDA and RLDA 
(1) TLDA

TLDA is the method that two path is prepared for transmitting data to the coordinator node. This method has an advantage of high responsibility and redundancy but has a risk of the data corrosion between both lines.

(2) RLDA

RLDA is a method of communicating alternately clockwise and counterclockwise. In this method the roles of transmitting and receiving are switched alternately one by one between lower number routing node and higher number routing node (ref. to Fig.7). As the result, the number of times of transmitting and receiving is averaged uniformity among all routing nodes.

\subsection{Comparison of LDA, TPDA and RLDA}

In this section I will describe the comparison of data traffic volume of each data acquisition method. The number of times of transmitting and receiving at the most busy routing node for each method in the case of 4 routing nodes exist as the example is shown in Fig.9. The number in a small box means the number of blocks of routing node (One block is corresponding to one small box in Fig.7) and the time is counted up to 32 packets arrival to the coordinator node. It is based on the real time communication concept which is realized under condition such the number of the blocks in one box in Fig.9 is equal to the number of total routing node. There are three kinds of data acquisition methods, RDA, TLDA and RLDA, here Liner Data Acquisition (LDA) is the method explained in Fig.7.
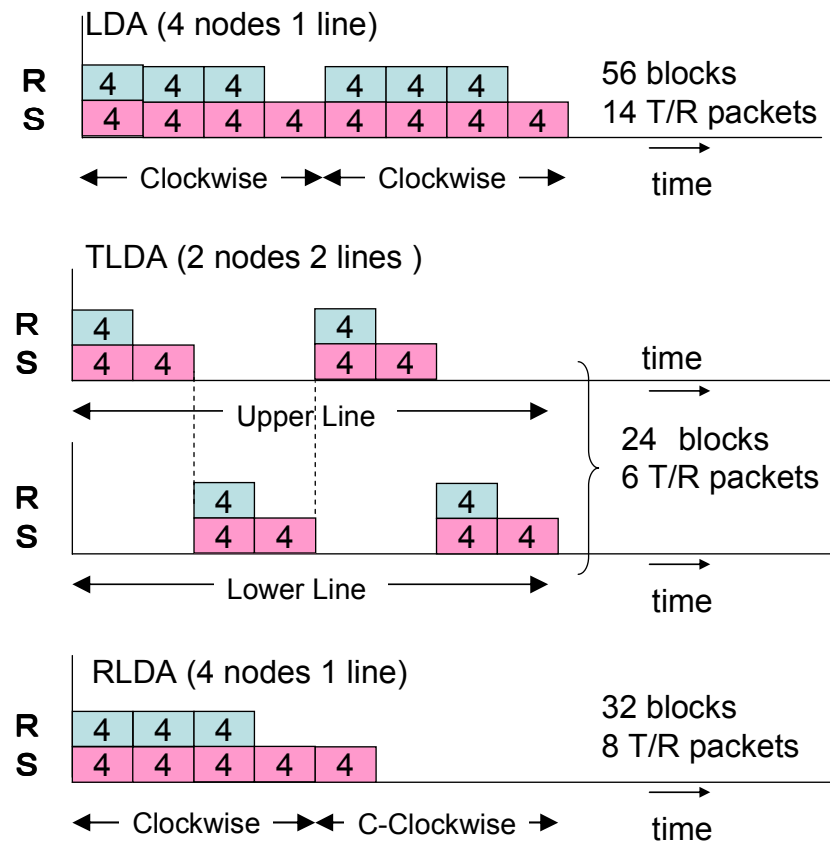

Fig.9. Data transmission on three kind of the method
Table.2. General Expression

\begin{tabular}{|c|c|c|}
\hline & Number of T/R & Normalized \\
\hline LDA & $2(2 n-1)$ & $2-1 / n$ \\
\hline TLDA(even) & $2(n-1)$ & $1-1 / n$ \\
\hline TLDA(odd) & $2 n$ & 1 \\
\hline RLDA & $2 n$ & 1 \\
\hline
\end{tabular}

As showing in Fig.9, TLDA is the smallest number of times of transmitting and receiving than other methods. Using the general expression in terms of $n$ (the number of routing node), total number of times of transmitting and receiving during $2 n$ packets ( $n$ (nodes) x 2 (times)) (8 packets in Fig. 9 example) arrives at coordinator from all routing nodes is shown in Table. 2 for each method. And normalized the numbers of times of transmitting and receiving per one packet are shown in the right column of Table 2.

TLDA has two expressions of $T / R$ times because the expressions are different when total node number is an odd or an even. Assuming the worst case where the total number becomes odd, TDLA and RDLA are regarded as having the same normalized $\mathrm{T} / \mathrm{R}$ times as 1 .

\section{Estimation of Network Scale}

When considering sensor network applying for HEMS in a house roughly the following range of conditions is assumed.

(i) Router number $\quad n=2,3,4,5$

(ii) End node number $\quad k=5,10,15,20$

(iii) Interval time $\quad d t$ (min.) $=\sim 5$

(iv) Method of data acquisition LDA, TDLA, RDLA

The total consumption average power $P$ is calculated as following in eq. (1).

$$
P=(p p \times p t+c p \times c t+s p \times s t) / d t
$$

Here $p p$ : packet power, $p t$ : packet time, $c p$ : CPU power, $c t$ : CPU time, $s p$ : sleep power, $s t$ : sleep time, $d t$ : duration time. Referring to Fig.5, Fig.6, Fig.7, Fig.9, the detailed parameter is obtained. We have defined total power for HEMS with energy harvesting as $20[\mathrm{uW}]$ described in section 2. Then the calculation is performed to derive the interval time to realize with the condition of routing node number $n$ and end node number $k$ and 20[uW] as the consumption average power applying to LDA and TLDA (odd), RLDA method. The calculated results applying to LDA are shown in Fig.10 and applying to TLDA (odd) or RLDA are shown in Fig. 11. 


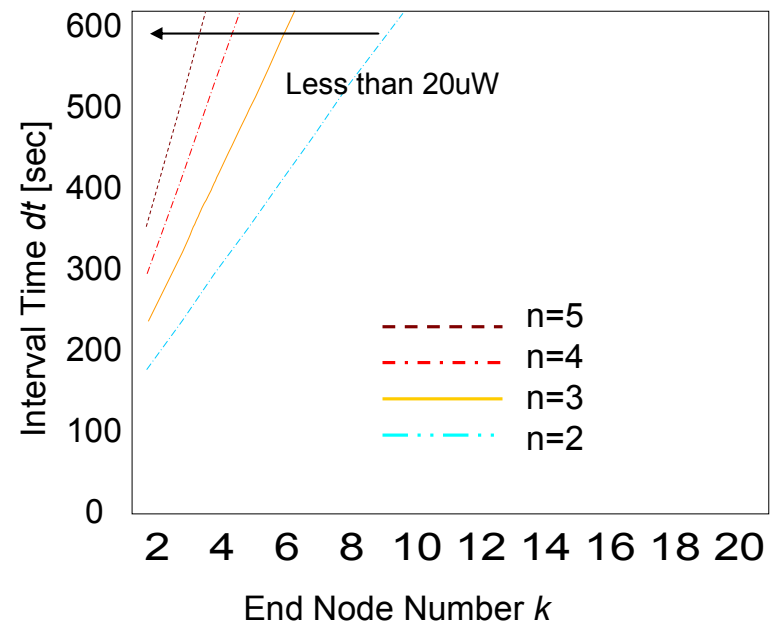

Fig.10. Estimation of Network Scale with LDA Method

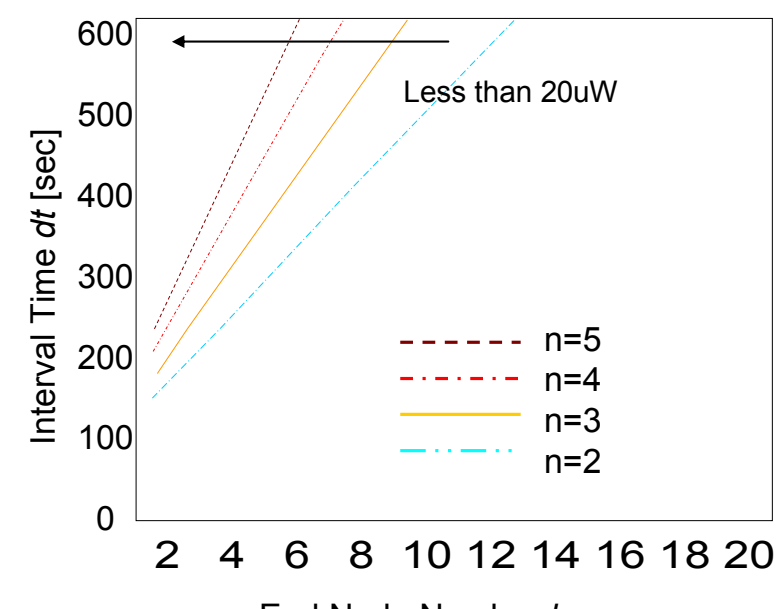

End Node Number $k$

Fig.11. Estimation of Network Scale with TLDA (odd) and RLDA Method

Extracting the data from the results in Fig.10 and Fig.11, we can find the maximum number of end nodes that can be implemented corresponding to the number of routing nodes. The summarized number is described in Tabe.3. Assuming ordinary scale of a house, 3 routing node and more than 7 end nodes is desirable. Referring to Table.3, when 3 routing nodes is used the end nodes can be used up to 6 with LDA method but 9 end nodes can be used with TLDA(odd) and RLDA method. As such example shows, the expandability of network by TLDA (odd) and RLDA method is very useful for HEMS or small area sensor network.

Table.3. Implementable Number of End Nodes

\begin{tabular}{|c|c|c|c|c|}
\hline Number of routing node & 2 & 3 & 4 & 5 \\
\hline LDA method & 9 & 6 & 4 & 3 \\
\hline TLDA (odd) and RLDA method & 12 & 9 & 7 & 6 \\
\hline
\end{tabular}

\section{Conclusion}

We have studied the sensor network HEMS. The merit of that study is the sensor nodes can be installed everywhere. On the other hand there two problems, one is based on battery supplier and the other is based on the transmitting rage restriction. The former one comes to be hopeful by our study for the energy harvesting verification experiments. In this study I seek for the ability to expand the communication field introducing routing nodes working by only the harvesting energy in a house. Assuming the transmitting range in a house, I focus about the line topology network. Defining the data time flow and the transmitting and receiving data sequence and the current consumption model, I estimated the network scale based on the HEMS specification that the average energy consumption is $20[\mathrm{uW}]$. In such estimation I think up to compare three ways of data acquisition method. As the calculation results, followings are clarified. In common Line Data Acquisition method (LDA), the network scale is smaller than the network scale in devised two methods, Two Line Data Acquisition (TLDA) and Rotated Data Acquisition (RLDA) in the condition of satisfying practical HEMS application. As the numerically result, the end node number in TLDA and RLDA is estimated as 6, twice of it in LDA when we use 5 routing nodes.

\section{References}

(1) Takashi Yoshikawa, "Novel Concept for HEMS Apparatus", Elsevier Science Direct 2012 Energy Procedia 14, pp.1273-1279, 2012.

(2) Thomas von Buren et al. ,"Optimization of Inertial Micro power Generators for Human Walking Motion", IEEE Sensors Journal, 6 (1), pp.28-38, 2006.

(3) Takashi Yoshikawa, "Many Kinds of Energy Source in Our Surroundings at Home", Springer Proceedings in Energy on ENEFM 2014, PP,307-312, 2015.

(4) Takashi Yoshikawa, Ikuo Awai, "A Novel Design for HEMS consisting of Sensor Network Nodes with Energy Harvesting and Wireless Power Transmission", Advances in Industrial Engineering and Management, Vol.2 No1 (2013),11-15. 